\title{
Influence d'un élevage ostréicole sur les flux de nutriments et d'oxygène dans un écosystème lagunaire
}

\author{
Nabila MAZOUNI, Jean-Marc DESLOUS-PAOLI, Sonia LANDREIN
}

Ifremer, laboratoire écologie conchylicole, BP 171, 1, rue Jean-Vilar, 34203 Sète cedex France

(Révisé le 7 juillet 1998, accepté le 20 juillet 1998)

\begin{abstract}
Impact of oyster culture on nutrients and oxygen fluxes in a coastal lagoon. The impact of suspended oyster culture (Crassostrea gigas, Thunberg) on oxygen and nutrient fluxes has been studied in situ, in a coastal lagoon (Thau, France), during a seasonal cycle. On the first plan of the multiple factorial correspondences analysis (MCA), seasons were well discriminated. The fluxes were maximum in summer and minimum in winter. However, this seasonal pattern was not only linked to the water temperature, as autumn and spring (similar temperatures of about $12{ }^{\circ} \mathrm{C}$ ) were distinct in the second factorial plan (2.3). Oxygen uptake by the oyster cultures varied between $0 \mu \mathrm{mol} \mathrm{m} \mathrm{m}^{-2} \mathrm{~h}^{-1}$ (January) and $11823 \pm$ $377 \mu \mathrm{mol} \mathrm{m} \mathrm{m}^{-2} \mathrm{~h}^{-1}$ (July). Ammonia and nitrate-nitrites were released into the water column respectively at a rate of 2905 $\pm 327 \mu \mathrm{mol} \mathrm{m}{ }^{-2} \mathrm{~h}^{-1}$ and $891 \pm 88 \mu \mathrm{mol} \mathrm{m}^{-2} \mathrm{~h}^{-1}$ in the summer and $0 \mu \mathrm{mol} \mathrm{m}{ }^{-2} \mathrm{~h}^{-1}$ and $177 \pm 97 \mu \mathrm{mol} \mathrm{m}^{-2} \mathrm{~h}^{-1}$ in the cold season. During the summer, the nitrate-nitrites flux was about $20 \%$ of the total dissolved inorganic nitrogen production. Phosphate rclease was low except for two periods during which an important release was measured; in May (1686 \pm 44 $\left.\mu \mathrm{mol} \mathrm{m}{ }^{-2} \mathrm{~h}^{-1}\right)$ and in November $\left(2691 \pm 800 \mu \mathrm{mol} \mathrm{m}^{-2} \mathrm{~h}^{-1}\right)$. No linear relation between water temperature and phosphate flux was found. In Thau Lagoon, oyster cultures (oysters and epibiota) by producing $2 \times 10^{7}$ mol- $\mathrm{N} \mathrm{y}^{-1}$ play a central role in nitrogen renewal in the water column. $\mathcal{C}$ Elsevier, Paris
\end{abstract}

oyster culture / fluxes / nutrients / oxygen

Résumé - L'influence d'un élevage d'huîtres creuses (Crassostrea gigas, Thunberg) sur les flux de nutriments et d'oxygène dans un écosystème lagunaire a été mesurée in situ au cours d'un cycle annuel. Une analyse factorielle des correspondances multiples ( $\mathrm{ACM}$ ) a permis de caractériser les quatre saisons en référence à la variabilité des flux observée au cours de la période d'étude. La consommation d'oxygène varie entre $0 \mu \mathrm{mol} \mathrm{m}^{-2} \mathrm{~h}^{-1}$ (janvier) et $11823 \pm 377 \mu \mathrm{mol} \mathrm{m}^{-2} \mathrm{~h}^{-1} \mathrm{en}$ juillet. L'ammonium et les nitrate-nitrites sont produits dans la colonne d'eau avec respectivement des valeurs maximales de $2905 \pm 327 \mu \mathrm{mol} \mathrm{m} \mathrm{m}^{-2} \mathrm{~h}^{-1}$ et $891 \pm 88 \mu \mathrm{mol} \mathrm{m}^{-2} \mathrm{~h}^{-1}$. En période hivernale, les flux de nitrate-nitrites sont nuls et ceux d'ammonium sont minimaux $\left(177 \pm 97 \mu \mathrm{mol} \mathrm{m}^{-2} \mathrm{~h}^{-1}\right)$. Par contre, en saison chaude, les nitrate-nitrites constituent une fraction importante $(20 \%)$ de l'azote inorganique dissous total produit dans la colonne d'eau. Les flux de phosphate sont orientés vers une production dans la colonne d'eau qui présente un pic en mai $\left(1686 \pm 44 \mu \mathrm{mol} \mathrm{m}{ }^{-2} \mathrm{~h}^{-1}\right)$ et un autre en novembre ( $\left.2691 \pm 800 \mu \mathrm{mol} \mathrm{m}^{-2} \mathrm{~h}^{-1}\right)$. Enfin, dans les secteurs conchylicoles, les résultats montrent que les élevages exercent une grande influence sur la régénération de l'azote dans la colonne d'eau. La présence d'une épibiose importante contribue, dans une certaine mesure, à augmenter cette influence. En effet, dans ces secteurs, les tables à huîtres produiraient en moyenne $2 \times 10^{7}$ mol d'azote par an, ce qui représente un impact très supérieur à celui obtenu antérieurement sans considerer pas les épibiontes. (1) Elsevier, Paris

élevage ostréicole / flux / nutriments / oxygène 


\section{INTRODUCTION}

Le rôle des populations de filtreurs dans le contrôle de l'équilibre des écosystèmes marins semi-fermés a été souligné dans de nombreux travaux $[2,7,12,16]$. L'introduction artificielle et la culture d'une biomasse élevée de coquillages peut être à l'origine de modifications importantes dans les échanges de matière au sein de l'écosystème. En effet, les filtreurs par leur mode alimentaire, renforcent la liaison entre les compartiments particulaire et dissous [10] et accélèrent les transferts de la matière organique vers les sédiments où elle s'accumule $[3,22,39]$. Les mollusques filtreurs contrôlent l'eutrophisation du milieu par leur broutage $[1,34]$, et participent au renouvellement du stock phytoplanctonique en apportant, par leur excrétion dissoute, des nutriments azotés et phosphorés $[9,14,16,38,39,42]$.

La lagune de Thau est un écosystème peu profond dans lequel la conchyliculture occupe une place importante. Cette activité produit chaque année, environ $15000 \mathrm{t}$ de coquillages. De plus, l'immersion continue des structures d'élevage entraîne le développement d'une abondante épibiose sur les cordes à huîtres $[25,28,32]$. Or, la présence en quantités élevées de ces assemblages d'organismes hétérotrophes (huîtres et épibiontes) dans la colonne d'eau est susceptible de modifier les transferts et la transformation de la matière au sein de cet écosystème lagunaire [27]. Malgré les nombreux travaux sur les filtreurs, peu d'études ont été réalisées sur des populations à partir d'incubations in situ $[6,7,38]$. Seuls Dame et Patten [11] et Dame et al. [13] utilisent un système in situ fonctionnant en flux continu (BEST) appliqué directement à des cultures d'huîtres et de moules disposées à plat en milieu intertidal et Outin [35] développe dans la lagune de Thau un système de raceway fonctionnant pour des huîtres posées à plat dans le milieu naturel.

Dans le présent travail, le système des enceintes métaboliques (Metabolic Enclosure System, MES) est utilisé afin de quantifier les échanges entre les élevages conchylicoles ct la colonne d'eau. Cette méthode est directement applicable aux structures d'élevage et fonctionne en flux continu (circulation d'eau) et en incubation. Un suivi in situ des variations saisonnières des flux de nutriments et d'oxygène à l'interface eau élevage est réalisé. L'objectif est de quantifier l'influence des élevages sur le recylage de ces éléments dans la colonne d'eau.

\section{MATÉRIEL ET MÉTHODES}

\subsection{Site étudié}

Le bassin de Thau est une lagune méditerranéenne salée de 7500 ha, situee au sud-ouest de la France (figure 1). Cette lagune côtière semi fermée présente un temps de renouvellement des eaux très long (trois à quatre mois) [31]. Les caractéristiques principales de la lagunc sont résumées dans le tableau 1 . Depuis le début du siècle, d'importantes activités conchylicoles y ont été développées [18]. Étendue sur 1500 ha, la conchyliculture occupe le cinquième de la superficie totale du bassin. Elle est répartie en trois secteurs : A, B et C (figure 1). La zone B est intermédiaire entre une zone $A$ profonde et riche en phytoplancton [23], où la croissance des animaux est la plus importante, et une zone $C$, plus pauvre, où les taux de croissance sont plus lents [17].

Les huîtres sont cultivées sur des cordes suspendues dans la colonne d'eau. Les coquilles collectrices, chargées de naissain originaire des côtes atlantiques françaises (bassins d'Arcachon ou de Marennes-Oléron), sont fixées entre les brins composant la corde. L'immersion continue des cordes à huîtres a pour conséquence la formation d'une importante épibiose. L'unité d'élevage considérée dans notre travail appelée « pigne » est constituée par un assemblage multispécifique ct composé par des huîtres et des épibiontes.

Un suivi mensuel de l'interface eau-élevage a été réalisé entre les mois de janvier et de décembre 1992. Les mesures ont été réalisées sur des pignes adultes de la zone B à $3 \mathrm{~m}$ de profondeur. Cette profondeur a été retenue afin d'obtenir une épibiose composée en majorité d'organismes hétérotrophes. En effet, la colonisation par les macrophytes, limitée principalement au premier mètre des cordes, est peu représentative de l'épibiose fixée sur l'ensemble de la corde.

\subsection{Méthodologie utilisée}

Les enceintes métaboliques (figure 2) sont constituees de deux hémisphères en métacrylate assujetties sur un arceau de PVC et dans lesquelles sont suspendus les animaux à étudier. Le volume total d'une enceinte métabolique est de 35,7 L. L'homogénéisation de l'eau est assurée par deux agitateurs magnétiques. Un courant d'eau cst produit au travers de l'enceinte par une pompe péristaltique située sur le bateau, selon un débit préalablement défini. Trois enceintes métaboliques avec des 


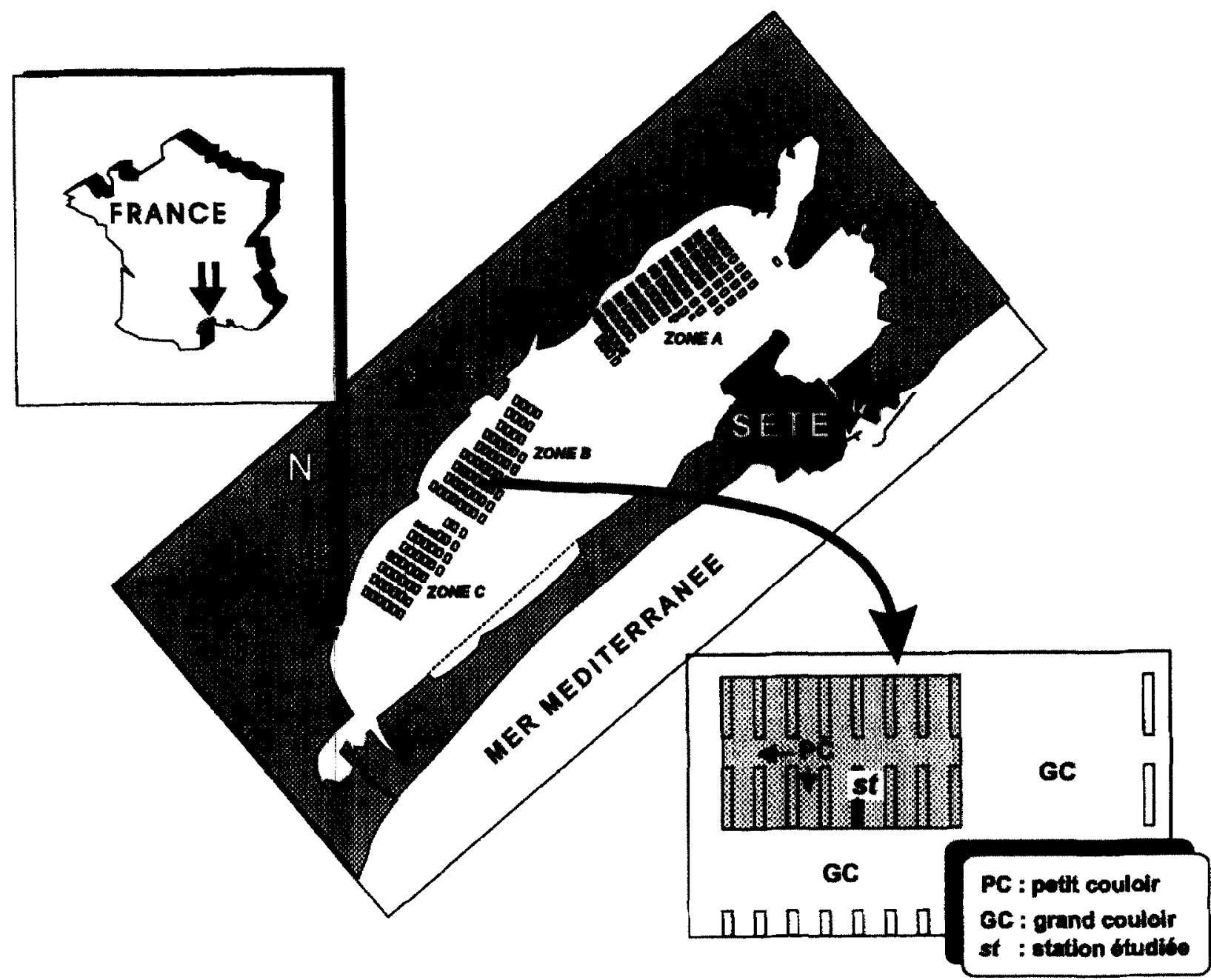

Figure 1. Situation géographique de la lagune de Thau et localisation des secteurs conchylicoles A, B et C et de la station étudiée.

Figure 1. Geographical situation of the Thau Lagoon and location of farming zones A, B, and C and the studied station.

animaux et une enceinte témoin vide sont utilisées pour chaque série de mesures. Les mesures débutent après $2 \mathrm{~h}$ de mise en équilibre (stabilisation) du système expérimental. Le système peut fonctionner en flux continu ou en incubation par simple arrêt de la pompe et fermeture des entrées d'eau en plongée. Dans les expériences, on considère que l'enceinte métabolique témoin reflète les caractéristiques de l'eau extérieure, donc de l'eau qui entre dans les enceintes expérimentales.

\subsection{Mesure des fonctions de respiration et d'excrétion}

Pour chaque expérience, l'évolution des concentrations en oxygène dissous et en nutriments dans les enceintes expérimentales (i.e. contenant une pigne) est comparée à celle de l'enceinte témoin. Les échanges d'oxygène sont mesurés lorsque le système fonctionne en incubation, grâce à des sondes polarographiques YSI 58 (étalonnées à l'air à $100 \%$ de saturation et selon la méthode de Winkler) qui enregistrent la concentration de l'oxygène en continu. La durée du confinement varie selon la saison de façon à ce que la consommation d'oxygène ne soit jamais supérieure à $30 \%$. En effet, en conditions hypoxiques, certaines especes sont susceptibles de modifier leur métabolisme respiratoire $[40,41]$. Parallèlement, dans chaque enceinte mélabolique, $60 \mathrm{~mL}$ d'eau sont prélevés à la seringue en plongée deux fois par heure pour l'analyse des éléments nutritifs. L'eau est filtrée sur Whatman 
Tableau I. Caractéristiques principales de la lagune de Thau.

Table I. Main characteristics of the Thau Lagoon.

\begin{tabular}{ll}
\hline Longueur : & $19,5 \mathrm{~km}$ \\
Largeur : & $4,5 \mathrm{~km}$ \\
Profondeur moyenne : & $4 \mathrm{~m}$ \\
Volume total : & $3010^{8} \mathrm{~m}^{3}$ \\
Températures extrêmes entre 1991 et 1994: & 3 et $25^{\circ} \mathrm{C}$ \\
Salinités extrêmes entre 1991 et $1994:$ & 28 et 40 \\
Chlorophylle $a:$ & 0,5 à $5 \mu \mathrm{g} \mathrm{L}^{-1}$ \\
Surface hors des zones conchylicoles : & 6000 ha \\
Surface des zones conchylicoles : & 1500 ha \\
Surface recouverte par les tables & 150 ha \\
Surface d'une table : & $6000 \mathrm{~m}^{2}$ \\
\hline
\end{tabular}

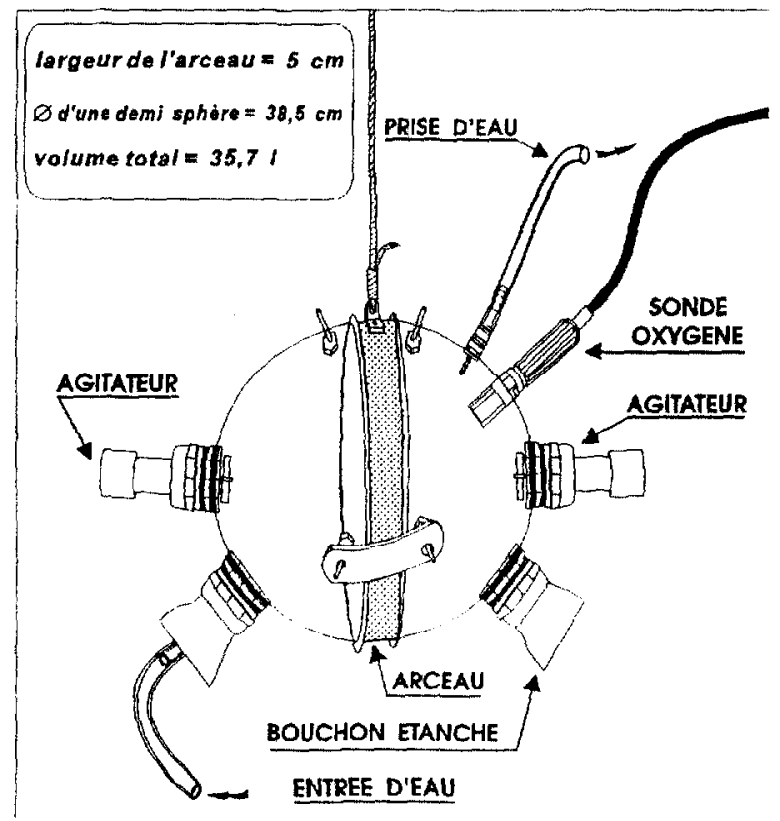

Figure 2. Schéma de détail d'une enceinte métabolique.

Figure 2. Structure of a metabolic enclosure.

GF/C, l'échantillon pour le $\mathrm{NH}_{4}^{+}$est fixé directement à bord tandis que l'eau nécessaire à l'analyse des autres nutriments est congelée. Les dosages du $\mathrm{NH}_{4}^{+}$ont été effectués selon la méthode de Koroleff [24]. Les autres nutriments sont dosés avec un autoanalyseur Alliance EV2 selon la méthode de Treguer et Le Corre [45].

Pour les trois enceintes expérimentales, les flux d'oxygène et de nutriments sont estimés par la pente de la régression linéaire établie entre la concentration de l'élément considéré et le temps d'incubation. Les valeurs négatives indiquent une consommation, les valeurs posi- tives une production dans la colonne d'eau. Les flux obtenus pour une unité d'élevage (pigne) sont ensuite exprimés en $\mu \mathrm{mol} \mathrm{m} \mathrm{m}^{-2} \mathrm{~h}^{-1}$. Au niveau d'une table ostréicole $\left(600 \mathrm{~m}^{2}\right)$, un nombre moyen de 1200 cordes sont suspendues. Chaque corde est en moyenne composée de 15 pignes. On peut donc considérer un nombre moyen de 30 pignes par $\mathrm{m}^{2}$ de sédiment. Ce changement d'unité a été possible après que l'homogénéité des trois pignes utilisées pour chaque expérience ait été vérifiée (Anova, $p<0,05$ ). Cette opération présente l'avantage de rendre comparable nos résultats avec ceux obtenus par d'autres auteurs $[7,9,12,13,14]$ et ceux obtenus pour l'interface eau-sédiment [29].

\subsection{Analyse des données}

Une analyse factorielle des correspondances multiples (ACM) a été réalisće afin d'obtenir une description synthétique de la dynamique de l'écosystème. L'ACM utilise un tableau disjonctif complet basé sur des variables codées et permet donc de prendre en considération des variables hétérogènes contenant des valeurs négatives. Dans lc cas présent, l'ACM a été misc en æuvre sur un tableau croisant des mois avec des valeurs de flux et de concentration des différents éléments (oxygène et nutriments). Chaque variable est codée en classes qui correspondent à des niveaux de flux ou de concentration ( 1 = faible, $2=$ moyen, 3 = fort, etc. $)$. Le détail des variables utilisées est donné dans le tablcau II.

\section{RÉSULTATS}

\subsection{Cycle annuel de variation des concentrations dans l'eau extérieure}

Qu'elle que soit la saison, le pourcentage de saturation en oxygène est toujours supérieur à $90 \%$, avec un maximum de $140 \%$ en mars 1992 (figure 3). Les concentrations d'ammonium varient entre $0,08 \mu \mathrm{mol} \mathrm{L}{ }^{-1}$ (printemps) et $2,5 \mu \mathrm{mol} \mathrm{L}^{-1}$ en hiver, avec un pic en automne de $9 \mu \mathrm{mol} \mathrm{L} \mathrm{L}^{-1}$ (figure 4). La concentration de phosphate dans l'eau est minimale au printemps $\left(0,31 \mu \mathrm{mol} \mathrm{\textrm {L } ^ { - 1 } )}\right.$ et maximale en août $\left(3,3 \mu \mathrm{mol} \mathrm{L}^{-1}\right)$ (figure 5). Pour cet élément, l'évolution saisonnière des concentrations semble suivre celle de la température (figure 6).

\subsection{Cycle annuel des flux}

Les flux résultants d'oxygène sont négatifs tout au long de l'année car les phénomènes de consommation prédo- 
Tableau II. Variables utilisées dans l'analyse factorielle des correspondances multiples : nomtenclature, nombre, amplitude et codes des classes définies.

Table II. Nomenclature, range and number of clusters defined for each variable used in the MCA.

\begin{tabular}{|c|c|c|c|c|}
\hline Variable de milieu & Nomenclature & Amplitude & Classe & Code \\
\hline $\begin{array}{l}\text { Température } \\
\text { (celsius) }\end{array}$ & TEMP & $\begin{array}{l}4 \text { à } 9 \\
9 \text { à } 18 \\
18 \text { à } 22 \\
22 \text { à } 26\end{array}$ & $\begin{array}{l}1 \\
2 \\
3 \\
4\end{array}$ & $\begin{array}{l}\text { TEMP1 } \\
\text { TEMP2 } \\
\text { TEMP3 } \\
\text { TEMP4 }\end{array}$ \\
\hline $\begin{array}{l}\text { Oxygène } \\
\text { (concentration en } \mathrm{mL} \mathrm{L}^{-1} \text { ) }\end{array}$ & $\mathrm{OO}_{2}$ & $\begin{array}{l}4 \text { à } 6,4 \\
6,4 \text { à } 10\end{array}$ & $\begin{array}{l}1 \\
2\end{array}$ & $\begin{array}{l}\mathrm{OO} 21 \\
\mathrm{OO} 22\end{array}$ \\
\hline $\begin{array}{l}\text { Ammonium } \\
\text { (concentration en } \mu \mathrm{mol} \mathrm{L}^{-1} \text { ) }\end{array}$ & $\mathrm{ONH}_{4}$ & $\begin{array}{l}0 \text { à } 0.25 \\
0.25 \text { a } 1 \\
\text { |à } 10\end{array}$ & $\begin{array}{l}1 \\
2 \\
3\end{array}$ & $\begin{array}{l}\text { ONH41 } \\
\text { ONH42 } \\
\text { ONH43 }\end{array}$ \\
\hline $\begin{array}{l}\text { Phosphate } \\
\text { (concentration en } \mu \mathrm{mol} \mathrm{L} \mathrm{L}^{-1} \text { ) }\end{array}$ & $\mathrm{OPO}_{4}$ & $\begin{array}{l}0,2 \text { à } 0,7 \\
0,7 \text { à } 4\end{array}$ & $\begin{array}{l}1 \\
2\end{array}$ & $\begin{array}{l}\text { OPO41 } \\
\text { OPO42 }\end{array}$ \\
\hline \multirow[t]{2}{*}{$\begin{array}{l}\text { Nitrate-nitrite } \\
\text { (concentration en } \mu \mathrm{mol} \mathrm{L}^{-1} \text { ) }\end{array}$} & ONO & $\begin{array}{l}0 \text { à } 0,9 \\
0,29 \text { à } 1\end{array}$ & $\begin{array}{l}1 \\
2\end{array}$ & $\begin{array}{l}\text { ONO231 } \\
\text { ONO232 }\end{array}$ \\
\hline & & 1 à 4 & 3 & ONO233 \\
\hline \multicolumn{5}{|l|}{ Variable de flux } \\
\hline $\begin{array}{l}\text { Oxygène } \\
\mathrm{ml} \mathrm{m}^{-\cdots} \mathrm{h}^{-1}\end{array}$ & $\mathrm{O}_{2}$ & $\begin{array}{l}-50 \text { à }-25 \\
-25 \text { à } 0 \\
0 \text { à } 70\end{array}$ & $\begin{array}{l}1 \\
2 \\
3\end{array}$ & $\begin{array}{l}\mathrm{O} 21 \\
\mathrm{O} 22 \\
\mathrm{O} 23\end{array}$ \\
\hline $\begin{array}{l}\text { Ammonium! } \\
\qquad \mu \mathrm{mol} \mathrm{m}^{-2} \mathrm{~h}^{-1}\end{array}$ & $\mathrm{NH}_{4}$ & $\begin{array}{l}-5 \text { à } 10 \\
10 \text { à } 40 \\
40 \text { à } 120\end{array}$ & $\begin{array}{l}1 \\
2 \\
3\end{array}$ & $\begin{array}{l}\mathrm{NH} 41 \\
\mathrm{NH} 42 \\
\mathrm{NH} 43\end{array}$ \\
\hline 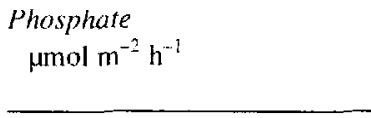 & $\mathrm{PO}_{4}$ & $\begin{array}{l}-20 \text { à } 0 \\
0 \text { à } 20 \\
20 \text { à } 120 \\
\end{array}$ & $\begin{array}{l}1 \\
3 \\
3 \\
\end{array}$ & $\begin{array}{l}\mathrm{PO} 41 \\
\mathrm{PO} 42 \\
\mathrm{PO} 43 \\
\end{array}$ \\
\hline \multicolumn{5}{|l|}{$\begin{array}{l}\text { Variables caractérisant } \\
\text { le compartiment « élevage " }\end{array}$} \\
\hline $\begin{array}{l}\text { Poids toial des pignes } \\
\text { (g) }\end{array}$ & РТOT & $\begin{array}{l}5 \text { à } 25 \\
25 \text { à } 45 \\
45 \text { à } 200\end{array}$ & $\begin{array}{l}1 \\
2 \\
3\end{array}$ & $\begin{array}{l}\text { PTOT1 } \\
\text { PTOT2 } \\
\text { PTOT3 }\end{array}$ \\
\hline $\begin{array}{l}\text { Poids toral hutres } \\
\text { (g) }\end{array}$ & PHUIT & $\begin{array}{l}0 \text { à } 10 \\
10 \text { à } 19 \\
19 \text { à } 40\end{array}$ & $\begin{array}{l}1 \\
2 \\
3\end{array}$ & $\begin{array}{l}\text { PHUIT } \\
\text { PHUIT2 } \\
\text { PHUIT3 }\end{array}$ \\
\hline $\begin{array}{l}\text { Poids toral épibiontes } \\
(\mathrm{g})\end{array}$ & PEPIB & $\begin{array}{l}0 \text { à } 15 \\
15 \text { à } 25 \\
25 \text { à } 100\end{array}$ & $\begin{array}{l}1 \\
2 \\
3\end{array}$ & $\begin{array}{l}\text { PEPIB } 1 \\
\text { PEPIB } 2 \\
\text { PEPIB } 3\end{array}$ \\
\hline
\end{tabular}

minent (figure 7), à l'exception du mois de novembre durant lequel l'oxygène est produit vers la colonne d'eau. Dès le début du printemps (mars), la consommation augmente et passe de $4685 \pm 2822 \mu \mathrm{mol} \mathrm{m} \mathrm{m}^{-2} \mathrm{~h}^{-1}$ à $8512 \pm$ $2230 \mu \mathrm{mol} \mathrm{m}{ }^{-2} \mathrm{~h}^{-1}$ (juin) et atteint en juillet un maximum de $11823 \pm 377 \mu \mathrm{mol} \mathrm{m} \mathrm{m}^{-2} \mathrm{~h}^{-1}$. En hiver, la consommation est réduite avec un minimum mesuré de $4138 \pm 1096$ $\mu \mathrm{mol} \mathrm{m}^{-2} \mathrm{~h}^{-1}$ à $8{ }^{\circ} \mathrm{C}$. Au mois de janvier, Iorsque la température avoisine $4^{\circ} \mathrm{C}$, aucun flux n'a été délecté.
Entre les mois de mars et de décembre, le phosphate est fourni à la colonne d'eau alors que durant l'hiver, aucun flux n'a été mesuré ifigure 8). Deux pics de production se distinguent dans le cycle annuel : un premier apparait au mois de mai ( $\left.1686 \pm 44 \mu \mathrm{mol} \mathrm{m}^{-2} \mathrm{~h}^{-1}\right)$, un autre au mois de novembre $\left(2691 \pm 800 \mu \mathrm{mol} \mathrm{m} \mathrm{m}^{-2} \mathrm{~h}^{-1}\right)$.

La production d'ammonium par les élevages est maximale en été $\left(2905 \pm 327 \mu \mathrm{mol} \mathrm{m}{ }^{2} \mathrm{~h}^{-1}\right)$ et minimale en 
hiver: $\left(177 \pm 97 \mu \mathrm{mol} \mathrm{m}^{-2} \mathrm{~h}^{-1}\right)$ (figure 9). Des flux de nitrate-nitrite ont également été mesurés avec un maximum estival de $891 \pm 88 \mu \mathrm{mol} \mathrm{m}^{-2} \mathrm{~h}^{-1}$, et un flux nul en période froide. En saison chaude, les flux de nitratenitrite représentent jusqu'à $20 \%$ du flux total d'azote inorganique dissous.

L'analyse des correspondances multiples a permis d'identifier les principales sources de variabilité des flux. Les trois premiers axes d'inertie, qui représentent $50 \%$ de la variabilité totale (tableau $/ I I$ ), sont retenus dans notre analyse. L'axe 1, défini par le poids total des pignes (PTOT) et le flux d'ammonium (NH4), met en évidence une opposition entue l'hiver et les autres saisons (figure 10). En hiver, les faibles températures s'accompagnent d'un poids total de pigne faible et de faibles flux d'ammonium. L'été et l'automne sont en opposition sur l'axe 2, défini essentiellement par le flux d'oxygène $\left(\mathrm{O}_{2}\right)$ et la température (TEMP). Par contre, l'effet de la température n'apparaît pas sur l'axe 3 qui oppose le printemps à l'automne. La période printanière semble définic par des concentrations importantes en oxygène dissous et des concentrations faibles en phosphate et en ammonium. Enfin, la bonne homogénéité des réplicats est aussi confirmée par l'analyse puisqu'ils présentent des projections voisines sur les axes factoriels de l'ACM. L'examen du second plan factoriel $(2,3)$ met en évidence l'existence

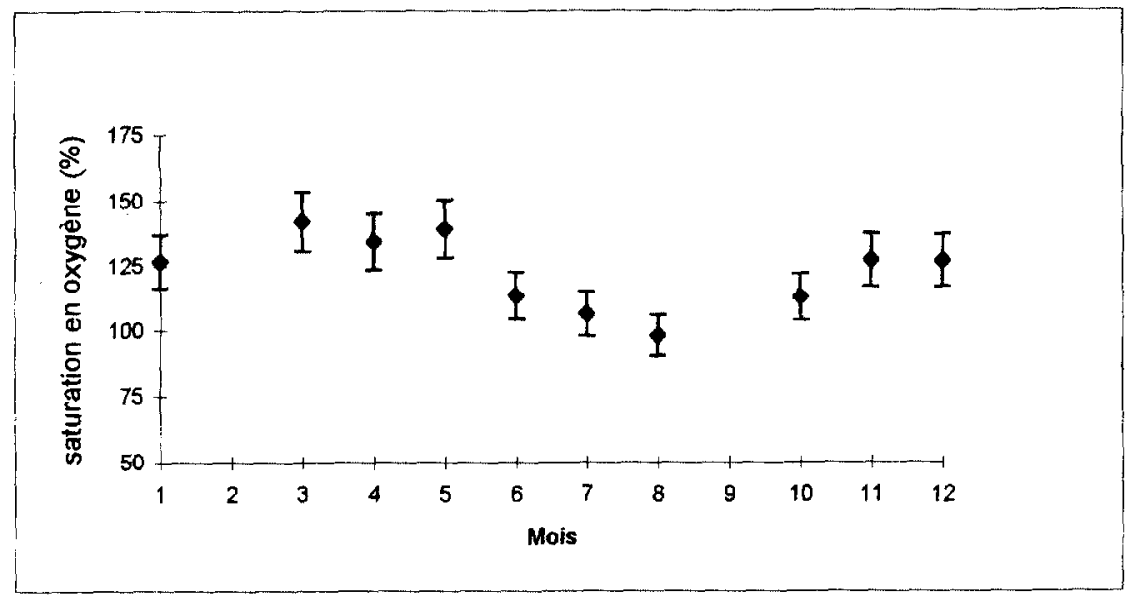

Figure 3. Variation du pourcentage de saturation des eaux en oxygène au cours d'un cycle annucl.

Figure 3. Annual variation of the percentage of saturation of oxygen in the water column.

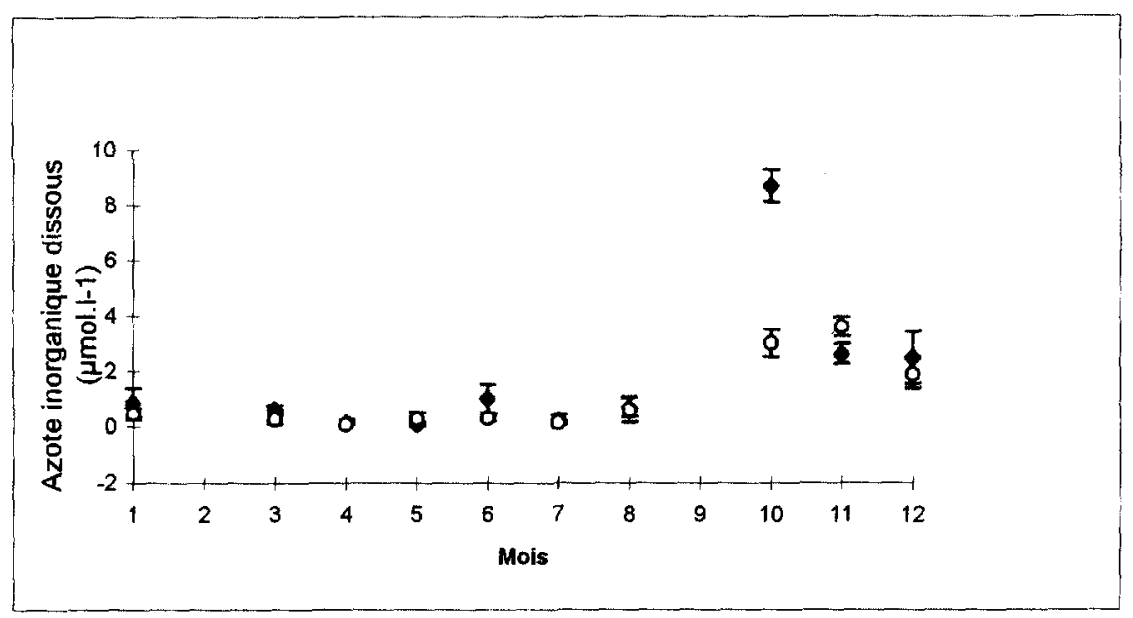

Figure 4. Évolution saisonnière des concentrations $\left(\mu \mathrm{mol} \mathrm{L}^{-1}\right)$ d'azote inorganique dissous dans la colonne d'eau. - : concentration d'ammonium, 0 : concentration de nitrate-nitrites

Figure 4. Seasonal variation of dissolved inorganic nitrogen $\left(\mu \mathrm{mol} \mathrm{L}{ }^{-1}\right)$ in the water column. $\bullet:$ ammonium, 0 : nitrates-nitrites. 


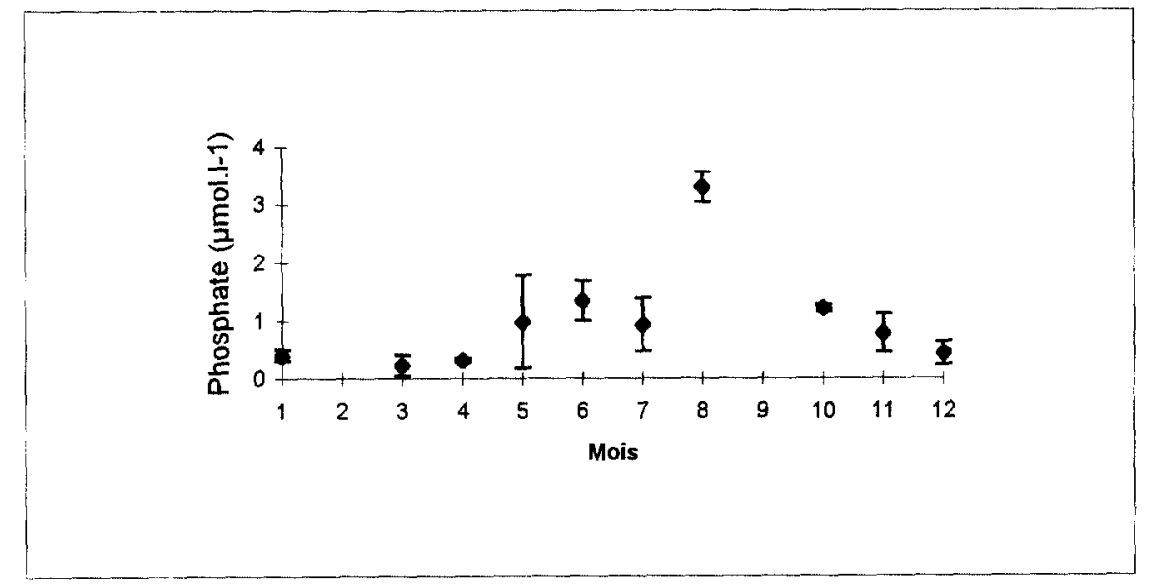

Figure 5. Évolution saisonnière des concentrations $\left(\mu \mathrm{mol} \mathrm{L} \mathrm{L}^{-1}\right)$ de phosphate dans la colonne d'eau.

Figure 5. Seasonal variation of phosphate concentrations in the water column $\left(\mu \mathrm{mol} \mathrm{L}{ }^{-1}\right)$.

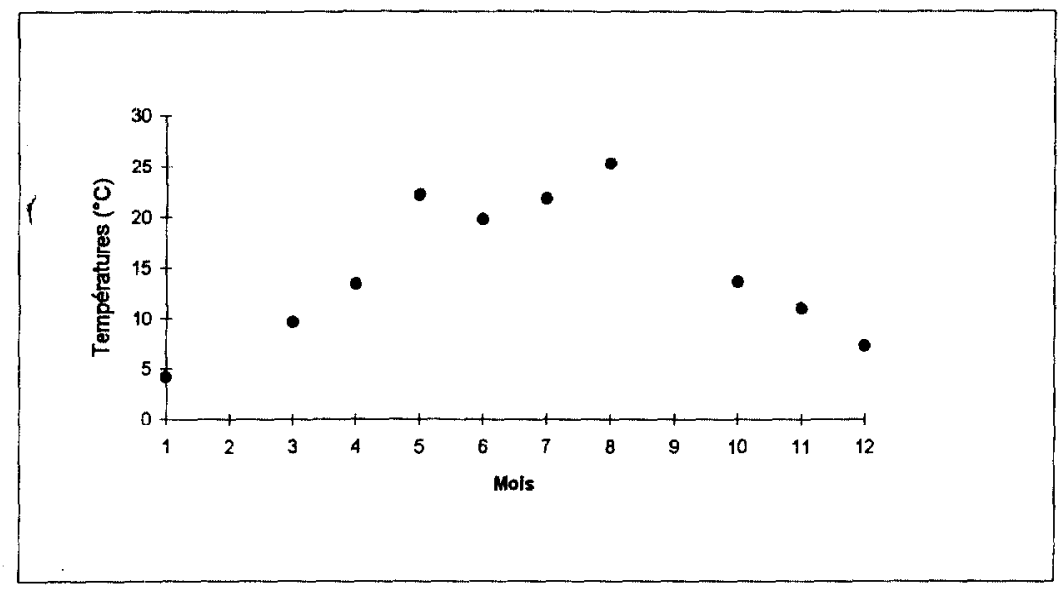

Figure 6. Évolution de la température de l'eau $\left({ }^{\circ} \mathrm{C}\right)$ au cours du cycle annuel.

Figure 6. Seasonal evolution of the water column temperature $\left({ }^{\circ} \mathrm{C}\right)$.

d'un gradient non linéaire pour certaines variables, comme la température, le flux d'ammonium et le flux de phosphate (figure II).

\section{DISCUSSION}

\section{1. Évolution des concentrations et des flux d'oxygène}

L'oxygène est un facteur d'équilibre du milieu [19] et sa consommation est une mesure de l'activité hétérotrophe d'un écosystème [15]. Les concentrations d'oxygène mesurées dans la colonne d'eau, correspondent à celles mesurées par Tournier et al. [43], et indiquent l'existence d'une certaine autotrophie de l'écosystème lagunaire. L'oxygénation de l'eau est assurée par une activité importante des producteurs primaires et par un brassage actif des masses d'eau sous l'effet du vent [44]. L'abondante couverture algale des sédiments [20] peut aussi expliquer les très bonnes conditions d'oxygénation des eaux. La consommation d'oxygène enregistrée dans notre travail est la résultante de la respiration des huîtres en élevage (C. gigas), de celle de leur épibiose, de l'activité bactérienne et de la demande chimique. Dans le cas de températures très basses, de l'ordre de $4{ }^{\circ} \mathrm{C}$ (janvier 1992), aucune mesure de flux n'a été obtenue, car l'ensemble 


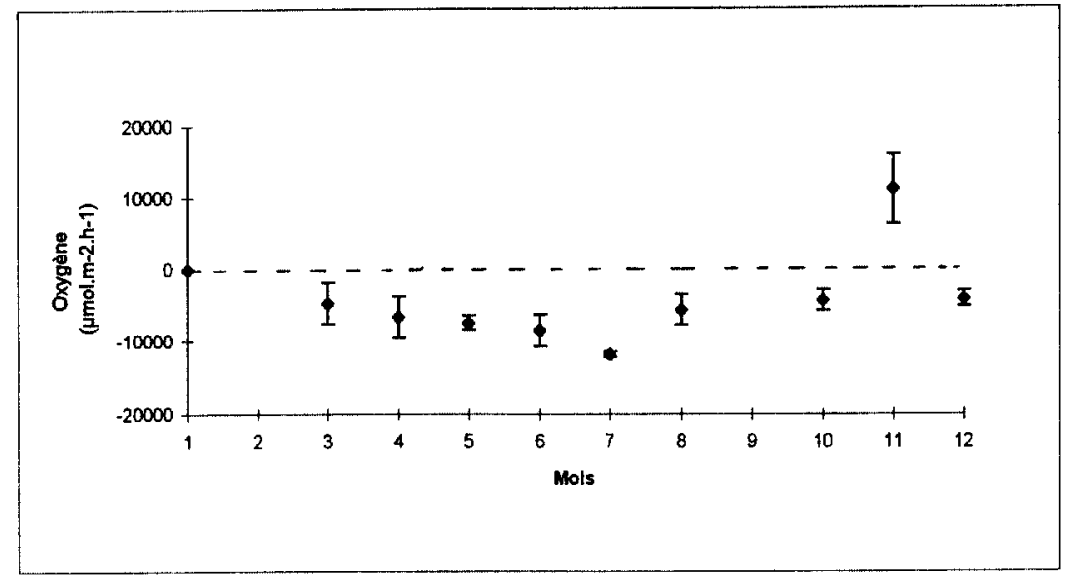

Figure 7. Variations saisonnières du flux d'oxygène $\left(\mu \mathrm{mol} \mathrm{m} \mathrm{m}^{-2} \mathrm{~h}^{-1}\right)$ à l'interface eau-élevage dans la zone conchylicole B.

Figure 7. Seasonal evolution of oxygen fluxes $\left(\mu \mathrm{mol} \mathrm{m}{ }^{2} \mathrm{~h}\right.$ ') due to the activities of the reared species in farming zone B in the Thau Lagoon.

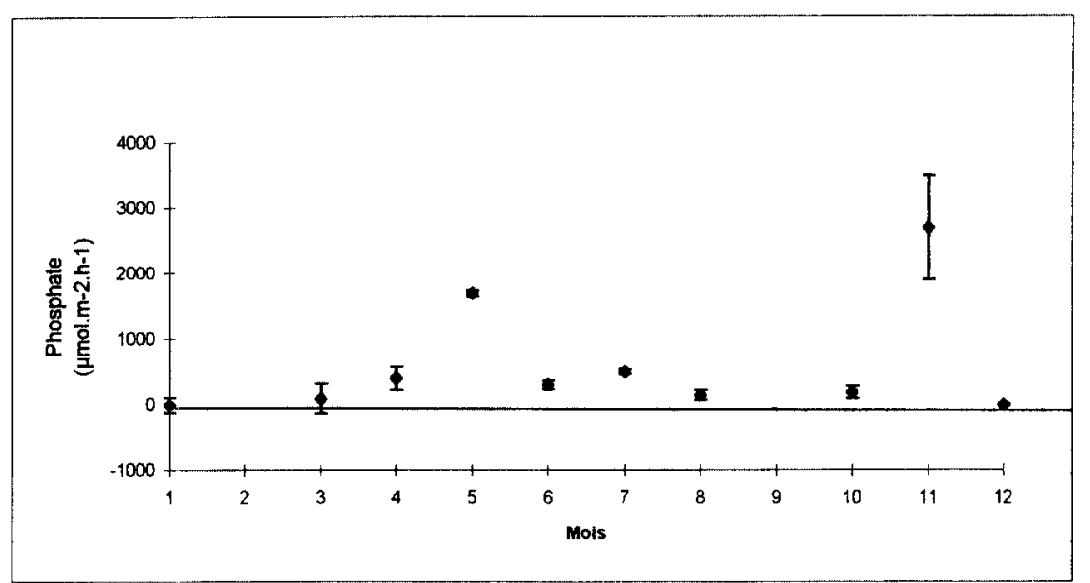

Figure 8. Variations saisonnières du flux de phosphate $\left(\mu \mathrm{mol} \mathrm{m}^{-2} \mathrm{~h}^{-1}\right)$ à l'interface eau-élevage dans la zone conchylicole B.

Figure 8. Seasonal evolution of phosphate fluxes $\left(\mu \mathrm{mol} \mathrm{m}^{-2} \mathrm{~h}^{-1}\right)$ due to the activities of the reared species in farming zone $B$ in the Thau Lagoon.

des animaux est en état de «vie ralentie » (paralysie ciliaire). En effet, la température est un des facteurs principaux de variation de la consommation d'oxygène $[4,5$, 37, 41]. Toutefois, l'absence de mesure de flux à cette période peut aussi indiquer que l'intensité très faible des échanges est située en dessous des limites de détection de la méthodologie utilisée.

Les flux négatifs d'oxygène que nous avons obtenus sont voisins de ceux enregistrés par Zuburg et Smaal [48] pour un banc de Inoules avec leur épifaune $\left(-10819 \mu \mathrm{mol} \mathrm{O}_{2}\right.$ $\mathrm{m}^{-2} \mathrm{~h}^{-1}$ ) et de ceux de Dankers et al. [15] qui indiquent des valeurs comprises entre -3360 et $-7840 \mu \mathrm{mol} \mathrm{O} \mathrm{O}_{2} \mathrm{~m}^{-2} \mathrm{~h}^{-1}$.
Contrairement aux travaux de Shumway [41] et de Outin [35], nous n'observons pas de diminution de la demande en oxygène pendant la période de ponte. Cependant, étant donné que nous mesurons un flux résultant, ce phénomène peut avoir été masqué par l'activité d'un autre groupe d'hétérotrophes.

\subsection{Phosphore}

Le phosphate est la forme du phosphore la plus excrétée par les mollusques. Il peut en constituer jusqu'à $74 \%$ [47]. Les valeurs des flux de phosphore obtenues ici sont 


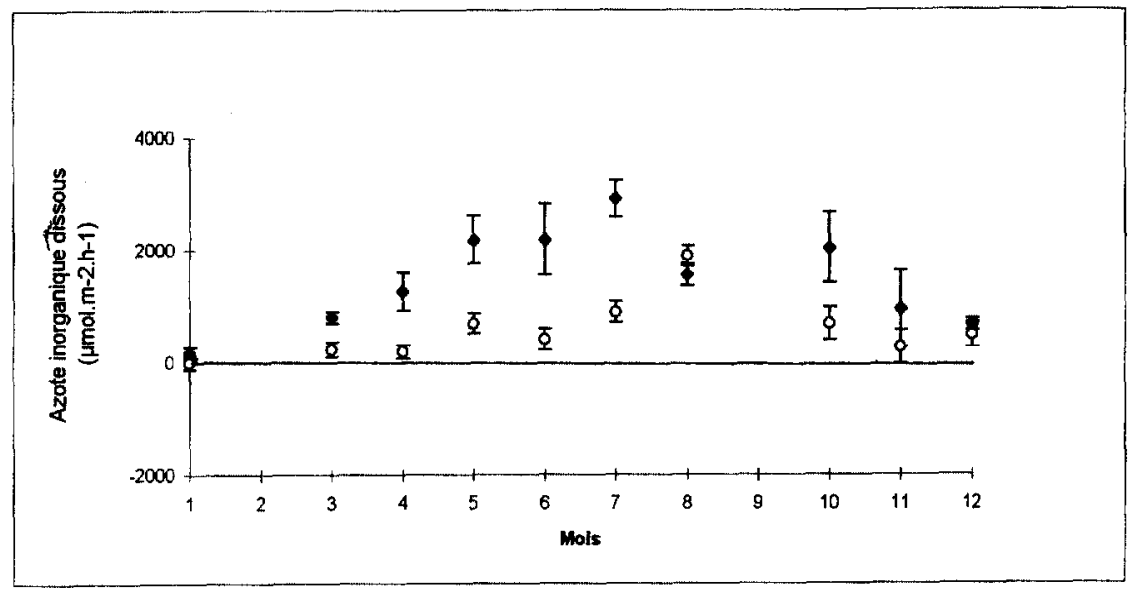

Figure 9. Variations saisonnières du flux d'azote inorganique dissous $\left(\mu \mathrm{mol} \mathrm{m} \mathrm{m}^{-2} \mathrm{~h}^{-1}\right)$ à l'interface eau-élevage dans la zone conchylicole B. : flux d'ammonium, o: flux de nitrate-nitrites.

Figure 9. Seasonal evolution of inorganic dissolved nitrogen fluxes $\left(\mu \mathrm{mol} \mathrm{m} \mathrm{m}^{-2} \mathrm{~h}^{-1}\right)$ due to the activities of the reared species in the farming zone $\mathrm{B}$ in the Thau Lagoon. $\bullet$ ammonia fluxes, o: nitrate-nitrites fluxes.

similaires à celles obtenues par Smaal [42] pour un banc de moules $\left(645 \mu \mathrm{mol} \mathrm{m}{ }^{-2} \mathrm{~h}^{-1}\right.$ en avril et $81 \mu \mathrm{mol} \mathrm{m}^{-2} \mathrm{~h}^{-1}$ en septembre) et par Prins et Smaal [39] pour la même espèce $\left(640 \mu \mathrm{mol} \mathrm{m}^{-2} \mathrm{~h}^{-1}\right.$ en avril et $240 \mu \mathrm{mol} \mathrm{m}^{-2} \mathrm{~h}^{-1}$ en juin). Malgré les très fortes variations liées à celles du cycle de la marée [12], nos résultats s'accordent avec la valeur moyenne donnée par Zuburg et Smaal [48] pour un banc de $C$. gigas en zone intertidale $\left(570 \mu \mathrm{mol} \mathrm{m} \mathrm{m}^{-2} \mathrm{~h}^{-1}\right)$. Cependant, du mois d'octobre au mois de décembre, la valeur des flux mesurés dans la lagune de Thau est proche de zéro, alors que pour Zuburg et Smaal [48], cette période se distingue dans le cycle saisonnier par des flux de phosphore significatifs, avec un relargage de l'ordre de $950 \mu \mathrm{mol} \mathrm{m} \mathrm{m}^{-2} \mathrm{~h}^{-1}$.

Le maximum de production de phosphate dans la colonne d'eau, observé entre la fin du printemps et l'automne, a déjà été décrit dans la littérature pour un banc de bivalves [12]. Selon ces auteurs, les sédiments situés sous les bancs d'huîtres seraient en grande partie responsables des flux enregistrés. Dans notre étude, il est probable que le flux de phosphate soit augmenté par des phénomènes de désorption du phosphore (réduction des oxydes et hydroxydes ferriques) qui apparaissent dans les microniches hypoxiques de la pigne, comme cela a été démontré dans le sédiment [30].

\subsection{Azote}

L'azote étant l'élément limitant de la production primaire dans la lagune de Thau [46], il était primordial d'en iden-
Tableau III. Résultats des calculs des pourcentages d'inertie expliquée par chacun des quatre premiers axes factoriels de l'ACM.

Table III. Percentages of the total inertia explained by the first four axes of the MCA (Multiple Correspondence Analysis).

\begin{tabular}{lccc}
\hline Axe factoriel & $\begin{array}{c}\text { Pourcentage } \\
\text { d'inertie } \\
\text { expliquée }\end{array}$ & $\begin{array}{c}\text { Pourcentage } \\
\text { cumulé }\end{array}$ & Valeur propre \\
\hline Axe 1 & 18,8 & 18,8 & 0,36 \\
Axe 2 & 17,8 & 36,6 & 0,32 \\
Axe 3 & 13,6 & 50,2 & 0,24 \\
Axe 4 & 7,6 & 57,8 & 0,15 \\
\hline
\end{tabular}

tifier les principales sources. Les flux d'ammonium que nous avons obtenus sont comparables à ceux de Dame et Dankers [10] qui ont travaillé sur un banc de moules en zone intertidale, mais sont supérieurs à ceux mentionnés par Nowicki et Nixon [33], Boucher et Boucher-Rodoni [6] et Lerat et al. [26]. Le maximum de production d'ammonium est observé en saison chaude (juillet 1992), période durant laquelle l'activité des animaux en élevage est maximale. Ce maximum de production d'ammonium est, par contre, bien inférieur aux valeurs moyennes recueillies par Dame et al. [12] pour un banc de Crassostrea virginica $\left(5500 \mu \mathrm{mol} \mathrm{m}^{-2} \mathrm{~h}^{-1}\right)$, par Dame et al. (1991) pour un banc de moules $\left(6071 \mu \mathrm{mol} \mathrm{m}^{-2} \mathrm{~h}^{-1}\right)$ ou encore au maximum de $13540 \mu \mathrm{mol} \mathrm{m} \mathrm{m}^{-2} \mathrm{~h}^{-1}$ enregistré pour des moules [39]. Les fortes densités de bivalves considérées par ces auteurs expliquent en partie ces flux importants. En effet, ces densités varient entre $900 \mathrm{~g} \mathrm{~m}^{-2}$ [14] et 


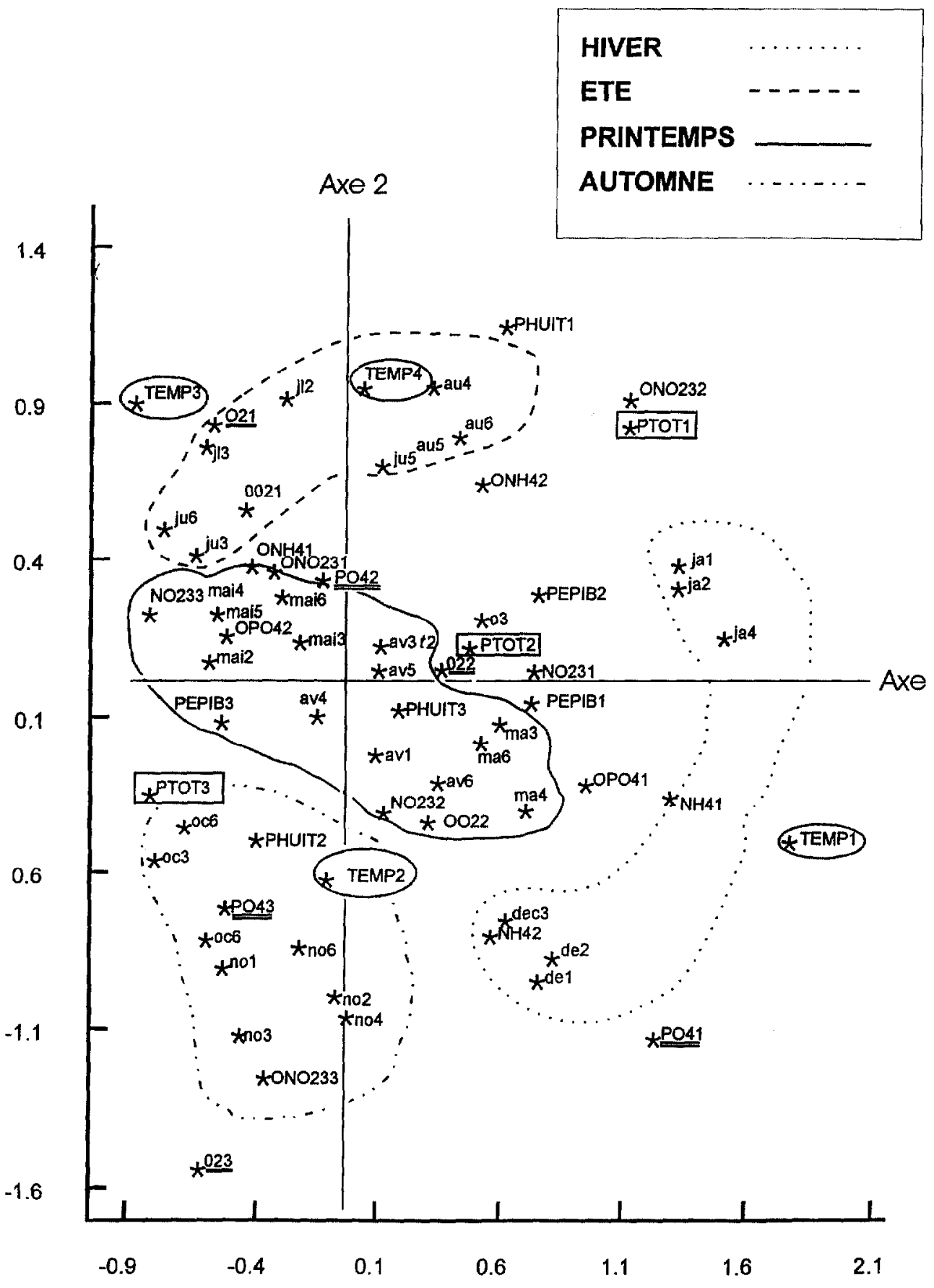

Figure 10. Représentation graphique du plan factoriel $(1,2)$ de l'analyse factorielle des correspondances multiples. (ja $=$ janvier, ma $=$ mars, ap $=$ avril, mai $=$ mai, $\mathrm{ju}=$ juin, $\mathrm{jl}=$ juillet, $\mathrm{au}=$ août, $\mathrm{oc}=$ octobre, no $=$ novembre, $d e=$ décembre) les numéros indiquent le numéro des enceintes métaboliques (réplicats). Le détail des variables est donné dans le tableau III.

Figure 10. Graphic representation of the first plan (1.2) of the multiple factorial analysis. $(\mathrm{ja}=\mathrm{January}, \mathrm{ma}=\mathrm{March}, \mathrm{ap}=\mathrm{April}$, mai $=\mathrm{May}$, $\mathrm{ju}=\mathrm{June}, \mathrm{jl}=\mathrm{July}, \mathrm{au}=$ August, $\mathrm{oc}=$ October, no $=$ November, $\mathrm{de}=$ December $)$. The numbers correspond to the replicates. The labels of the variables are detailed in table $I I$. 


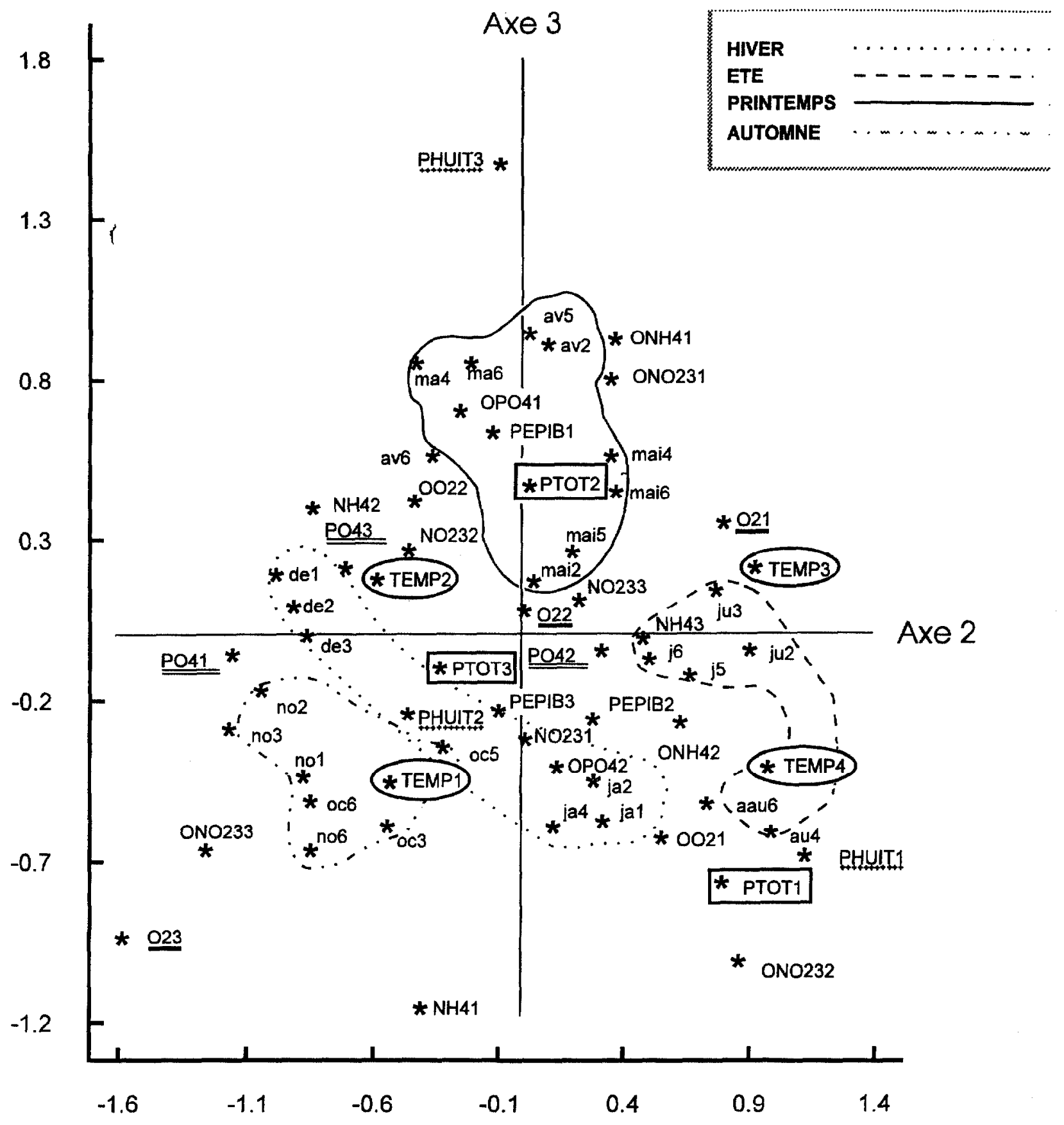

Figure 11. Représentation graphique du plan factoriel $(2,3)$ de l'analyse factorielle des correspondances multiples. (ja $=$ janvier, ma $=$ mars, ap $=$ avril, mai $=$ mai, $\mathrm{ju}=$ juin, $\mathrm{jl}=$ juillet, au $=$ août, $\mathrm{oc}=$ octobre, no $=$ novembre, de $=$ décembre) les numéros indiquent le numéro des enceintes métaboliques (réplicats). Le détail des variables est donné dans le tableau III.

Figure 11. Graphic representation of the second plan (2.3) of the multiple factorial analysis. (ja = January, ma $=$ March, ap $=$ April, mai $=$ May, $\mathrm{ju}=\mathrm{June}, \mathrm{jl}=\mathrm{July}, \mathrm{au}=$ August, $\alpha c=$ October, no $=$ November, $\mathrm{de}=$ December $)$. The numbers correspond to the replicates. The labels of the variables are detailed in table $I I$. 
$2200 \mathrm{~g} \mathrm{~m}^{-2}$ [39] de matière sèche sans cendre ; soit plus du double de celle considérée dans notre travail (1000 $\mathrm{g} \mathrm{min}^{-2}$ de mattière sèche totale).

Par ailleurs, ces auteurs travaillant sur des élevages posés à plat sur le sédiment, les valeurs importantes qu'ils obtiennent témoignent aussi de la contribution des sédiments au flux résultant. Ce phénomène a été décrit par Zuburg et sinaal [48] qui enregistrent pour les mêmes espèces et à biomasse équivalente, des flux d'ammonium de $4500 \mu \mathrm{mol} \mathrm{m}^{-2} \mathrm{~h}^{-1}$ quand les bivalves sont directement posés sur le fond, contre seulement $1200 \mu \mathrm{mol} \mathrm{m}^{2} \mathrm{~h}^{1}$ quand les animaux étudiés sont placés sur des tables audessus des sédiments. Ainsi, une partie importante de l'ammonium qui diffuse dans la colonne d'eau provient de la reminéralisation benthique [39]. Or, dans les secteurs conchylicoles, cette reminéralisation de l'ammonium est d'autant plus importante que les biodépôts sont abondants [8, 14].

La production de nitrate-nitrite par l'élevage que nous avons enregistrée en période estivale correspond à celles obtenues pour des élevages à plat sur les sédiments $[10$, $39,42]$. Ces productions importantes d'azote inorganique dissous à l'interface eau-élevage (trop élevées pour provenir du métabolisme des animaux) semblent pouvoir être en partie expliquées par la structure même du compartiment «élevage». En effet, l'accumulation entre les coquilles des huîtres et les épibiontes, de particules de matière organique (biodépôts) est à l'origine de la formation d'une vérilable interface eau-sédiment au sein du compartiment élevage [27] dans laquelle des polychaetes détritivores et des crustacés se développent. Les flux mesurés s'expliqueraient donc par l'activité des communautés présentes (microorganismes et macroorganismes) et par l'existence de phénomènes de nitrification et d'ammonification au niveau de ce compartiment sédiment suspendu. La réactivité très forte de ces sédiments situés sous les élevages a pu êtrc confirmée dans un récent travail [21].

La structure du compartiment élevage est done susceptible de modifier la dynamique des échanges dans la colonne d'eau. L'analyse factorielle des correspondances multiples (ACM) a permis de confirmer qu'outre le poids total des pignes, le poids en huîtres dans la pigne et le poids en épibiontes comptent parmi les principales sources de variabilité des flux. Par ailleurs, l'ACM a permis de décrire et de décomposer le cycle saisonnier. En termes de fonctionnement (flux), on distingue plusieurs situations : une opposition très marquée entre l'hiver et l'été et une situation intermédiaire concernant l'automne et le printemps. L'automnc et le printemps sont discriminés dans le plan factoriel $(2,3)$ grâce aux caractéristiques du milieu. Ainsi, on peut constater que l'influence saisonnière n'est pas exclusivement dépendante de la température. Cette analyse met également en évidence l'existence de relations non linéaires entre certaines variables, notamment entre le flux de phosphate et la température.

En conclusion, cette première approche sur l'influence des élevages sur les flux d'éléments nutritifs et d'oxygène met en évidence, non seulement l'effet de la température mais aussi celui de la biomasse en élevage et de la quantité de matière accumulée sur les pignes d'élevage. Les élevages assurent un recyclage efficace des nutriments azotés et en particulier de l'ammonium. En s'appuyant sur les travaux de Outin [35] sur des huîtres isolées de leur épibiose, Picot et al. [36], estiment que les élevages produiscnt par leur excrétion $10^{7} \mathrm{~mol}^{-1}$ pour l'ensemble de la lagune. En considérant l'élevage dans son ensemble, on obtient un flux d'ammonium moyen produit de $1500 \mu \mathrm{mol} \mathrm{m} \mathrm{m}^{-2} \mathrm{~h}^{-1}$ ce qui correspond à une excrétion de $2 \times 10^{7} \mathrm{~mol} \mathrm{an}^{-1}$. Ces résultats soulignent donc l'intérêt de considérer l'élevage dans sa totalité (huîtres et épibiontes) et de ne pas se limiter à la sculc espèce cultivée.

\section{Remerciements}

Les auteurs remercient Raphaël Plante, ainsi que les deux arbitres anonymes pour leurs commentaires. Sincères remerciements à l'ensemble de l'équipe du laboratoire DEL-Écologie de l'Ifremer de Sète pour sa collaboration. Ce travail a été réalisé grâce au soutien financier de I'Ifremer et du PNOC-Oxythau.

\section{RÉFÉRENCES}

[1] Alpine A.E., Cloern J.E., Trophic interactions and direct physical effects control phytoplankton biomass and production in an estuary, Limnol. Oceanogr. 37 (1992) 946-955.

[2] Asmus H.R., Asmus R.M., Phytoplankton-mussel bed interaction in intertidal ecosystems, in : Dame R.F. (ed.), Bivalves fil- ter feeders in estuarine and coastal ecosystem processes, NATO ASI Series, G 33, Berlin, 1993, 57-84.

[3] Baudinet D., Flux nutritifs particulaires et dissous dans un écosystème mytilicole côtier méditerranéen, thèse doct. univ. Aix-Marseille-II (1991) $136 \mathrm{pp}$. 
[4] Blanchard M., Analyse fonctionnelle et méthodologique pour l'étude bioénergétique de mollusques, rapp. Ifremer Brest. DRO-EL/ 89-18 (1989) $100 \mathrm{p}$

[5] Bougrier S., Geairon P., Deslous-Paoli J-M., Bacher C., Jonquière G., Allometric relatioship and effects of temperature on clearance and oxygen cusumption rates of Crassostrea gigas (Thunberg), Aquaculture, 134 (1995) 143-154

[6] Boucher G., Boucher-Rodoni R., In situ measurement of respiratory metabolism and nitrogen fluxes at the interface of oysters beds, Mar. Ecol. Prog. Ser. 44 (1988) 229-238.

[7] Boucher-Rodoni R., Boucher G., In situ study of the effect of oyster biomass on benthic metabolic exchange rates, Hydrobiologia 206 (1990) 115-123.

[7] Cloern J.E., Does the benthos control phytoplankton biomass in south San Francisco Bay?, Mar. Ecol. Prog. Ser. 9 (1982) 191-202.

[8] Dahlbäck B., Gunnarsson L.A.H., Sedimentation and sulfate reduction under a mussel culture, Mar. Bial. 63 (1981) 269 275

[9] Dame R.F., The role of bivalve filter feeder material fluxes in estuarine ecosystems, in: Dame R.F. (éd.), Bivalves filter feeders in estuarine and coastal ecosystem processes. NATO ASI Series, G 33, Berlin, 1993, 245-269.

[10] Darne R.F., Dankers N., Uptake and release of materials by a wadden sea mussel bed, J. Exp. Mar. Biol. Ecol. 118 (1988) 207-216.

[11] Dame R.F., Patten B.C.. Analysis of energy flows in an intertidal oyster reef, Mar. Ecol. Prog. Ser. 5 (1981) 115-124.

[12] Dame R.F., Spurrier J.D., Wolawer T.G., Carbon, nitrogen and phosphorus processing by an oyster reef, Mat. Ecol. Prog. Ser. 54 (1989) 249256.

[13] Dame R.F., Zingmark R.G., Haskin E., Oysters reef as a processor of estuarine materials, J. Exp. Mar. Biol. Ecol. 83 (1984) 239-247.

[14] Dame R., Dankers N., Prins T., Jongsma H., Smaal A., The influence of mussel beds on nutrients in the Western Sea and Eastem Scheldt estuaries, Estuaries 14 (2) (1991) 130-138,

[15] Dankers N., Dame R., Kersting K., The oxgen consumption of mussel beds in the Dutch Wadden Sea,. in Topics in Marine Biology, Ros J.D. (éd.), Scientia Mar. 52 (2-3) (1989) 473476

[16] Dame R.F., Libes $S$, Oyster reefs and nutrient retention in tidal creeks, J. Exp. Mar. Biol. Ecol. 17 (1993) 251-258.

[17] Deslous-Paoli J.-M., Mazouni N., Souchu P. Landrein S. Pichot P., Juge C., Oyster farming impact on the environment of a mediterranean lagoon, Preliminary results of the OXYTHAU program, in : Dame R.F. (éd.), Bivalves filter feeders in estuarine and coastal ecosystem processes. NATO ASI Series, G 33, Berlin, 1993, 519-521

[18] Doumenge F., L'exploitation des eaux du bassin de Thau, Revue de l'économie méridionale : bulletin trimestriel du centre régional de la productivité et des études économiques, 7 (27) (1959) 243-266.
[19] Fontevieille D., Les mesures de rcspiration et leur intérĉt pour l'écologje des milieux aquatiques, Rev. Fr. Sci. Eau 25 (1983) 367-387.

[20] Gerbal M., Analyse spatio-temporelle des peuplements phytobenthiques de substrat meuble de l'étang de Thau (Hérault, France), thèse doct. univ. Aix-Marseille-II (1994) 241 pp.

[21] Gilbert F., Souchu P., Bianchi M., Bonin P.. Influence of shellfish-farming activities on nitrification, nitrate reduction to ammonium and denitrification at the water sediment interface of the Thau lagoon, Mar. Ecol. Prog. Ser. 151 (1997) 143153.

[22] Grenz C., Quantification et destinée de la biodéposition en zones de production conchylicole intensive en Méditerranée, thèse doct. univ. Aix-Marseille-II (1989) $152 \mathrm{pp}$.

[23] Jarry V., Étude pluridisciplinaire en écologie lagunaire (étang de Thau, France) : stratégie d'échantillonnage et organisation spatiale du phytoplancton, thèse doct. univ. Montpellier-II (1990) $173 \mathrm{pp}$.

[24] Koroleff $F$, Direct determination of ammonia in natural waters as indophenol blue, Int. Counc. Explor. Sea, CMC : 9 (1969) $1-6$.

[25] Lamy N., Organisation, structure et dynamique des peuplements macrobenthiques d'une table conchylicole de l'étang de Thau (Hérault, France), thèse doct. univ. Montpellier-II, (1996) 292 p. + annexes.

[26] Lerat Y., Boucher G., Le Corre P., Échanges à l'interface eausédiment dans un secteur côtier à forte biodéposition (parc ostréicole): cas de l'ammoniaque et des nitrates. Cah. Biol. Mar. 26 (1985) 393-408

[27] Mazouni N., Gaertner J.-C., Deslous-Paoli J.-M., Influence of oyster culture on water colurnn characteristics in a coastal lagoon (Thau, France), Hydrobiologia, $373 / 374$ (1998) 149156.

[28] Mazouni N., Étude in situ de ]'influence des élevages ostréicoles sur le fonctionnement d'un écosystème lagunaire méditerraneén, thèse doct. univ. Aix-Marseille-II (1995) $256 \mathrm{p}$.

[29] Mazouni N., Gaertner J-C., Deslous-Paoli J-M.. Landrein S., Geringes d'Oedenberg M.. Nutrient and oxygen exchanges at the water-sediment interface in a shellfish farming lagoon (Thau, France), J. Exp. Mar. Biol. Ecol. 203 (2) (1996) 91113.

[30] Mesnage V., Contribution à l'étude de la mobilité des formes de phosphate à l'interface eau-sédiment dans des écosystèmes lagunaires, thèse doct. unjv. Montpellier-I (1994) 252 pp.

[31] Millet B. Fonctionnement hydrodynamique du bassin de Thau, validation d'un modèle numérique de circulation (programme Ecothau), Oceanol. Acta 12 (1989) 37-46.

[32] Monniot $F$. Mise en évidence d'un filtre " micropore » naturel chez les Ascidiacea, C.R. Acad. Sci. Paris 289 (1979) $927-$ 929.

[33] Nowicki B.L., Nixon S.W., Benthic community metabolism in a coastal lagoon ecosystem. Mar. Ecol. Prog. Ser. 22 (1985) $21-30$. 
[34] Officer C.B., Smayda T.J., Mann R., Benthic filter feeding : a natural eutrophication control, Mar. Ecol. Prog. Ser. 9 (1982) $203-210$.

[35] Outin V., Écophysiologie de l'huître Crassostrea gigas (Thunberg) en milieu naturel, rôle des populations d'une lagune méditerranéenne dans les transferts des matières particulaire et dissoute, thèse doct. univ. Paris-VI (1990) $130 \mathrm{p}$.

[36] Picot B., Pena G., Casellas C., Bondon D., Bontoux J., Interpretation of the seasonal variations of nutrients in a mediterrancan lagoon : ćtang de Thau, Hydrobiologia 207 (1992) 105 114.

[37] Piveteau F., Effet de la température et de l'état physiologique de $C$. gigas sur ses fonctions d'acquisition et de dépenses métaboliques, mém. IUT biologie appliquée, univ. Bordeaux (1989) $67 \mathrm{p}$.

[38] Prins T.C., Smaal A.C., Benthic -pelagic coupling: the release of inorganic nutrients by an intertidal bed of Mytilus edulis, in : Barnes M., Gibson R.N. (éd.), Trophic relationship in the marine environment, Aberdeen University Press, Aberdeen, $1989,1990,89-103$.

[39] Prins T.C., Smaal A.C., The role of the blue mussel Mytilus edulis in the cycling of nutrients in the Oostershelde estuary (The Netherlands), Hydrobiologia 282-283 (1994) 413-429.

[40] Riva A., Massé H., Étude écophysiologique de quelques mollusques bivalves, bases biologiques de l'aquaculture, Ifremer, actes de colloques, Montpellier, 1 (1985) 45-62.

[41] Shumway S.E., Oxygen consumption in oysters an overview, Mar. Biol. Letters 3 (1981) 1-23.
[42] Smaal A.C., The ecology and cultivation of mussels: ncw advances, Aquaculture 94 (1991) 245-261.

[43] Tournier H., Audabram D., Landrein S., Deslous-Paoli J.-M., L'oxygène en été dans l'étang de Thau, Contribution à l'étude du risque de « Malaïgue », rapp. Ifremer, Sète (1987) 27 pp.

[44] Tournier H., Deslous-Paoli J-M., Variations spatio-temporelles estivale de l'oxygène dans les secteurs conchylicoles de l'étand de Thau, in : Amiart J.-C., Robert J.-M. (éd.), Le littoral, ses contraintes environnementales et ses conflits d'utilisation, Isomer, univ. Nantes (1992) 121-124.

[45] Tréguer P., Le Corre P., Manuel d'analyses de sels nutritifs dans l'eau de mer, utilisation de l'autoanalyzer II Technicon, $2^{e}$ éd., Laboratoire d'océanographie chimique, univ. Bretagne occidentale, Brest (1975).

[46] Vaquer A., Troussellier M., Courties C., Bibent B., Standing stock and dynamics of picophytoplankton in the Thau Lagoon (northwest Mediterranean coast), Limnol. Oceanogr. 41 (8) (1996) 1821-1828.

[47] Vincendeau M.L., Robert J.M., Variations qualitatives saisonnières des produits excrétés par deux mollusques bivalves Crassostrea gigas (Thunberg) et Ruditapes philippinnarum (Adam et Reeve), Oceanis 13 (1987) 543-556.

[48] Zuburg W., Smaal A.C., Uptake and release of suspended and dissolved material by oyster and mussel beds in the bay of Marennes-Oléron (France), Report of the Directoraat-Generaal Rijkswaterstaat, The Netherlands (1993) 51 pp. 